\title{
LA INFORMACION Y LA POLITICA DE PERSONAL
}

\author{
POR \\ Vicente MARía González-HABa
}

\begin{abstract}
SUMARIO: I. El Registro Central de Personal.-ll. La importancia de la información.-III. El personal estatal.-IV. El personal autonómico.-V. El personal local.-VI. Reflexiones finales.
\end{abstract}

Como ha dicho Anicet Le Pors, la Administración Pública no debe ser contemplada tan sólo desde el prisma negativo de su inclinación al papeleo, el formalismo o la rutina, sino que es preciso estudiarla desde ángulos más positivos y favorables. En este sentido, el entonces Ministro francés de la Función Pública y de las Reformas Administrativas señalaba que «la Administración no está fatalmente abocada a la burocracia», sino que, por el contrario, es preciso valorarla desde tres nuevas perspectivas. En primer término, debe ser «el lugar privilegiado del desarrollo de técnicas avanzadas, de los métodos de gestión más sofisticados, de los avances sociales más decisivos». En segundo lugar, "es el dominio privilegiado para la puesta a punto de los métodos de gestión más elaborados» en las áreas de personal, presupuesto, contratación, toma de decisiones, etc. $Y$, finalmente, ha sido desde siempre el punto de ureferencia social en materia de salarios, empleos y derechos", ya que lo que la Administración hace y decide repercute inexorablemente sobre el resto de los agentes sociales (1).

Este protagonismo de la Administración en la vida social, con lo que conlleva de ejemplaridad y símbolo para los demás agentes que actúan en una colectividad determinada, conduce a que, cada vez más, se incremente el interés por el conocimiento de cuantos elementos conforman una Administración Pública, con especial atención hacia su elemento humano o personal.

En España, sin embargo, la aproximación a la burocracia pública desde posiciones no exclusivamente jurídicas ha sido aislada, insuficiente e imperfecta. Nos hemos preocupado más de estudiar el régimen jurídico de los funcionarios públicos que de abordarlos desde su vertiente sociológica, cultura, social, financiera, histórica, etcétera, con lo que hemos dejado en la penumbra aspectos que hoy se consideran esenciales; y entre los cuales figura destacadamente el del volumen de los efectivos públicos que sirven en las diferentes Administraciones Públicas.

(1) LE PORS, Anicet: «Discours de cloture», en "La Fonction Publique», Revue française d'Administration Publique, núm. 25, janvier-mars 1983, pp. 85-86. 


\section{EL REGISTRO CENTRAL DE PERSONAL}

Sin embargo, debe reconocerse que, desde hace unos años, estamos asistiendo a un cambio de dirección en los estudios sobre las Administraciones Públicas. Ya no se las enfoca exclusivamente con los criterios tradicionalmente jurídicos y formalistas, sino que se considera por los expertos que el enfoque, para ser útil y real, ha de caracterizarse por su globalidad, su sentido pluridisciplinar, su amplitud metodológica; de tal manera que el objeto de estudio, en este caso las Administraciones Públicas, no sea prisionero de una sola disciplina ni coto cerrado de los cultivadores de una ciencia determinada. Como ha recordado Beltrán Villalba, hoy las cosas son de distinta forma que antes "y los científicos sociales españoles se ocupan de la Administración Pública desde sus propios supuestos teróricos, no meramente con una actividad residual definida por lo que no es» (2).

Como decíamos más arriba, el conocimiento y cuantificación del personal del sector público pasa por ser, hoy, una magnitud que es preciso conocer a todos los efectos económicos, financieros, sociales, culturales, políticos, etc. No obstante, para que logremos acercarnos a un cálculo lo más certero posible del número de empleados públicos en un país determinado, es indispensable que se den simultáneamente tres circunstancias, por supuesto no siempre coincidentes ni fácilmente realizables. La primera es que por parte de los gestores del sector público exista una voluntad decidida y clara de conseguir unas estadísticas fiables que permitan conocer el volumen del personal que trabaja en dicho sector, poniendo fin a situaciones de incertidumbre y ambigüedad, en ocasiones interesadas y provocadas desde el propio poder, para ocultar a la sociedad hechos o realidades que no se quieren divulgar. La segunda es que los gestores públicos, además de la voluntad acabada de referir, utilicen los medios técnicos más apropiados para conseguir su objetivo de dimensionar, en sus variadas facetas, todas las burocracias públicas. $Y$ la tercera es que, dada la singular configuración del sector público disperso en multitud de organizaciones más o menos extensas, se requiere que entre todas ellas se asegure una coordinación fluida y permanente para el intercambio de datos, perfeccionamiento y contraste de los mismos, trasvase de información y recíproco apoyo de unas y otras.

A nuestro juicio, sólo cuando existan las tres premisas acabadas de exponer -voluntad política, empleo de nuevas tecnologias $y$

(2) Beltrán VILLALba, Miguel: «Sociología de la Administración Pública», en Sociología en España, de Salvador Giner y Luis Moreno (editores), Instituto de Estudios Sociales AvanzadosConsejo Superior de Investigaciones Científicas, Madrid, 1990, p. 107. 
coordinación entre las organizaciones- empezaremos a salir del "subdesarrollo" estadístico en que, durante muchos años, se ha movido de la Función Pública española. Los autores se han encargado de explicar esta insatisfactoria situación con referencia a España, al tiempo que, en sus exposiciones, han ido describiendo el alcance y validez de los estudios llevados a cabo en la materia. Asi, Junquera González, tras indicar que ha habido aportaciones recientes de cierto interés, señala que utodavía desconocemos en gran medida la dimensión real de nuestra función pública, sus orígenes sociales e intelectuales, los rasgos típicos de su estructura profesional, su movilidad geográfica y funcional, los perfiles ideológicos más típicos de este complejo grupo social y su incidencia en el mercado de trabajo, en el sistema educativo y en la economía en general» (3).

La cuestión de cuantificar adecuadamente el empleo en el sector público español ha estado irresuelta siempre entre nosotros 0 , si se quiere, resuelta pero insuficiente e inapropiadamente. Los datos ofrecidos no han sido de plena fiabilidad ni han estado respaldados por una garantía científica plena, al ser fruto de la investigación de personas o grupos que, pese a su excelente buena voluntad, han chocado siempre con el muro infranqueable de una Administración desconfiada y recelosa en descubrir sus usecretos» y con la dificultad insuperable de una carencia alarmante de estadísticas bien elaboradas y oportunamente divulgadas y coordinadas entre sí.

Un instrumento de capital interés para este tipo de actividades es el Registro Central de Personal, creado por la Ley 30/1984, de 2-de agosto, de Medidas para la Reforma de la Función Pública, cuyo artículo 13 establece que «en la Dirección General de la Función Pública existirá un Registro Central en el que se inscribirá a todo el personal al servicio de la Administración del Estado y en el que se anotarán preceptivamente todos los actos que afecten a la vida administrativa del mismo". En su apartado 2, que es de carácter básico, se señala que «las Comunidades Autónomas y las Entidades Locales constituirán también Registros de Personal», si bien «cuando las Entidades Locales no cuenten con suficiente capacidad financiera o técnica, las Comunidades Autónomas, por sí mismas o por delegación en las Diputaciones Provinciales, los Cabildos o los Consejos Insulares, cooperarán a la constitución de dichos Registros». Y el apartado 3, igualmente de naturaleza básica, ordena que "todos los Registros de todas las Administraciones Públicas estarán coordinados» (el subrayado es nuestro), por lo que uel Gobierno, a propuesta del Ministro de la Presidencia

(3). JunQuera GonzÁlez, Juan: La función pública en la "Europa de los Doce», Instituto Nacional de Administración Pública, Alcalá de Henares (Madrid), 1986, p. 33. 
(ahora para las Administraciones Públicas), y previa deliberación del Consejo Superior de la Función Pública, regulará los contenidos mínimos homogeneizadores de los Registros de Personal y los requisitos y procedimientos para su utilización recíproca» (el subrayado es nuestro) (4).

De este modo, el legislador, consciente de la importancia de los Registros de Personal para una gestión moderna, dinámica y actualizada del personal, apela en el artículo 13 de la Ley de Medidas, como se acaba de exponer, a dos ideas fundamentales, como son, de una parte, la de coordinación, ya que este principio organizativo esencial hoy para el buen funcionamiento del Estado de las Autonomías y de las diferentes piezas que componen este gran engranaje, lo es también en el ámbito de la informacion sobre personal, y de otra parte, la de reciprocidad, puesto que los Registros, una vez implantados y en funcionamiento, deben estar preparados para su aprovechamiento recíproco e integral que permita agilizar la gestión del personal cada vez más dotado de movilidad y con posibilidades crecientes de traspasar las fronteras de una Administración Pública para instalarse en otra. Por eso el legislador, con buen criterio tanto jurídico como meramente operacional, ha declarado básico este apartado 3 del artículo 13, según se explicita en el artículo 1.3 de la Ley de Medidas.

Quedaría incompleta esta breve exposición sobre la normativa del Rigistro de Personal si no se hiciera referencia, siquiera

(4) En la Administración Autonómica todas las Leyes de Función Pública, a excepción de la de Cantabria que sólo ordena que «todos los expedientes de personal quedarán integrados en el correspondiente Registron (artículo 63.2), regulan con mayor o menor amplitud el Registro de Personal. Dicha regulación, por lo general, abarca la ubicación orgánica del Registro, las limitaciones a la utilización de sus datos previstas en el artículo 18.4 de la Constitución, la necesidad de su coordinación con otros Registros, los datos a inscribir y la referencia a la percepción de nuevas retribuciones. Es interesante el contenido del artículo 25 de la Ley de 23 de julio de 1985, de la Función Pública de la Administración de la Generalidad, según el cual «el Registro General de Personal funciona informáticamente», añadiendo que "a tal efecto, será competencia de cada Departamento la introducción de los datos iniciales y el mantenimiento permanentemente actualizado de la información relativa a todo el personal que tenga adscrito, así como la protección del acceso al Registro».

En la Administración Local, en cuanto a los funcionarios con habilitación de carácter nacional la Ley reguladora de las bases de Régimen Local de 2 de abril de 1985, en su artículo 99.5. establece que, en la Administración del Estadon, se llevará un Registro relativo a los funcionarios locales con habilitación nacional, en el que deberán inscribirse, para su efectividad todas las incidencias y situaciones de dichos funcionarios». Por su parte, el Real Decreto 1174/1987, de 18 de septiembre, por el que se regula el régimen jurídico de los funcionarios con habilitación de carácter nacional, dice que el citado Registro «se llevará en el registro Central de Personal de la Administración del Estado, anotando en el mismo los hechos e incidencias a que hace referencia el Real Decreto 1405/1986, de 6 de junio».

Sobre los funcionarios propios de las Corporaciones Locales, establece la Ley 7/1985, de 2 de abril, reguladora de las bases del Régimen Local, que aquéllas «constituirán Registros de Personal, coordinados con los de las demás Administraciones Públicas, según las normas aprobadas por el Gobierno. Los datos inscritos en tal Registro determinarán las nóminas a efectos de la debida justificación de todas las retribuciones» (artículo 90.3). Precepto que debe completarse con lo que ordena la Ley de Medidas en su articulo $13.2 \cdot$ y 3 , ya comentados. 
sucintamente, a dos disposiciones reglamentarias de evidente interés. La primera es el Real Decreto 1405/1986, de 6 de junio, por el que se aprueban el Reglamento del Registro Central de Personal y las normas de coordinación con los de las restantes Administraciones Públicas, y dictado en cumplimiento de lo que preceptúa el artículo 13.1, párrafo segundo, de la Ley de Medidas, según el cual «el Gobierno, a propuesta del Ministro de la Presidencia (ahora para las Administraciones Públicas), aprobará las normas reguladoras del Registro Central y el programa para su implantación progresiva». Este Reglamento dedica su capítulo I al "Registro Central de Personal», regulando el personal que debe inscribirse en el mismo, actos objeto de inscripción, anotaciones sucesivas, confección de títulos, expedición de certificaciones, Centro Informático, etc. A su vez, el capítulo II, denominado "Coordinación del Registro Central de Personal y los de las restantes Administraciones Públicas», al tiempo que determina que «los contenidos homogeneizadores" de los Registros de Personal son los establecidos en los artículo 2 (personal a inscribir) y 4.1 (actos y resoluciones a inscribir del personal funcionario y laboral), dicta varias normas acerca de la constitución de los diversos Registros, acceso telemático al Registro Central de Personal, apoyo a prestar por la Dirección General de la Función Pública (ahora Dirección General de Organización, Puestos de Trabajo e Informática) y utilización recíproca de la información.

La segunda disposición, de rango reglamentario, que debe citarse aquí es el Real Decreto 221/1987, de 20 de febrero, por el que se determina la estructura orgánica básica del Ministerio para las Administraciones Públicas, y en cuyo artículo $4 .^{\circ}$, sobre las competencias de la Dirección General de Organización, Puestos de Trabajo e Informática, se establece en su apartado 4 que compete a esta Dirección General "el impulso y elaboración de procesos informáticos que afecten a la gestión de personal de la Administración del Estado; la gestión informatizada del Registro Central de Personal; la inscripción y anotación de todos los datos administrativos del personal al servicio de la Administración del Estado, así como la coordinación con los Registros de Personal de todas las Administraciones Públicas». De esta manera, al suprimirse el Registro Central de Personal que existía como Subdirección General incorporada a la Dirección General de la Función Pública, según señala la disposición adicional séptima del Decreto 221/1987, que suprime igualmente otras Unidades orgánicas y Centros directivos, se la incardina en la Dirección General de Organización, Puestos de Trabajo e Informática a través de una de las seis Subdirecciones 
Generales que la componen, concretamente de la de Proceso de Datos de la Administración Pública (5).

Con la legislación expuesta, ha quedado perfilado jurídicamente el Registro de Personal que, si bien hasta la fecha, no ha sido objeto de atención en la medida y con el interés deseados, para el futuro se deberá convertir en un mecanismo indispensable de gestión de los recursos humanos (GRH). Así se ha podido escribir que "el Registro Central de Personal, aparte de tener como objetivo la satisfacción de las necesidades de información que requiere una sociedad democrática, debe de servir también como un instrumento de apoyo a los responsables de la gestión de personal», de tal manera que se transforme en un elemento dinamizador $y$ agilizador de dicha política "devolviendo la información que suministran las unidades de personal, convenientemente estructurada, para que pueda ser útil a la gestión ordinaria de estas unidades administrativas».

Esta consideración del Registro de Personal, como un excelente dispositivo de ayuda a los responsables de la gestión de los recursos humanos públicos, requiere obviamente que el Registro se monte de manera que la información que reciba y suministre sea lo más amplia y completa posible. En la actualidad, en cada oficina delegada del Registro Central de Personal hay instalada una Base de datos que recoge los relativos al personal de que se trate, sea del Ministerio, Organismo autónomo, Delegación del Gobierno o Gobierno Civil. Dicha Base dispone de una estructura de información referente a datos del Registro Central de Personal, a datos de las Relaciones de Puestos de Trabajo y a datos del Centro Gestor de que se trate. Todos estos datos se interrelacionan en la Base de datos descentralizada $y$, a través de ellos, los gestores de personal pueden obtener la información que soliciten.

(5) Sobre el origen del Registro de Personal, debe citarse el articulo 12 de la Ley de Funcionarios Civiles del Estado de 7 de febrero de 1964 que estableció que, en la Secretaría General de la Comisión Superior de Personal «existirá un Registro de Personal al servicio de la Administración Civil del Estadon, atribuyendo al titular de dicha Secretaría General, en el artículo 20. "organizar y dirigir el Registro de Personal de la Administración Civil del Estado".

Posteriormente, la Orden de 7 de octubre de 1964 estableció el modelo al que habrian de ajustarse las relaciones de funcionarios pertenecientes a Cuerpos o Escalas de la Administración Civil del Estado y dictó normas para su confección. Por otra Orden de 5 de mayo de 1970 se dieron normas sobre recogida de datos en relación con los funcionarios de carrera de la Administración Civil del Estado. Y la Orden de 30 de julio de 1973 reguló el funcionamiento del Registro de Personal, mientras la Resolución de 19 de abril de 1974 determinó los impresos normalizados a utilizar en los actos y resoluciones inscribibles en aquél.

En todo caso, el Registro creado por la Ley de 1964 no siguió una trayectoria brillante en cuanto a su eficacia y utilidad, convirtiéndose en un instrumento previsto por el legislador pero, en la práctica, sin apenas virtualidad y sin aplicación real y efectiva. 
A su vez, la Base de datos mencionada está soportada en una red de área local, de tal modo que todas las unidades que se dedican a gestionar recursos humanos puedan acceder tanto a la Base de datos local como al Registro Central de Personal. Las razones de ello «son evidentes ya que, en múltiples circunstancias, no sólo se necesita el conocimiento de la situación actual del funcionario, sino también su historia administrativa, y ésta sólo puede recogerse en el Registro Central de Personal».

Quiere decirse, pues, que el Registro Central de Personal es, de un lado, un factor que contribuye decididamente a mejorar $y$ clarificar la gestión $y$, de otro, es un elemento esencial de información en cuanto que almacena, suministra y recoge los datos precisos para ello. Sin embargo, "no basta que el Registro de Personal tenga la información que la gestión de los recursos humanos necesita, sino que además es imprescindible el que el Registro de Personal esté gestionado con las técnicas más avanzadas que estén hoy disponibles» (6).

\section{LA IMPORTANCIA DE LA INFORMACION}

La información es un presupuesto básico sobre el que se asientan las sociedades democráticas. Los ciudadanos de los países en los que imperan los valores determinantes de un verdadero Estado de Derecho reclaman, cada vez con mayor intensiciad, de las instancias gubernamentales que actúen en la gestión de los asuntos públicos con honestidad, con eficacia, con dedicación; pero también les solicitan que, en todos sus actos, brille la transparencia informativa. Los ciudadanos, los administrados, los usuarios, los vecinos quieren conocer lo que hacen, y cómo lo hacen, sus gestores públicos para luego entrar a valorar los resultados de su actividad y aceptarla o rechazarla a través de su opinión manifestada a través de las urnas.

Pero si el hombre de la calle pide crecientemente que se le informe sobre el destino del dinero que paga a través de los impuestos, sobre la defensa del medio ambiente donde vive $y$ trabaja, sobre las posibilidades educacionales y profesionales de sus hijos, sobre la urbanización de su ciudad o su barrio, también, a la inversa, la Administración Pública demanda de la sociedad la adecuada información que necesita para acertar en la toma de sus decisiones. Por ello, cada vez tiende a convertirse en más participa-

(6) «Los sistemas de gestión de información sobre personal en las Administraciones Públicas», Secretaría General Técnica del Ministerio para las Administraciones Públicas, Madrid, 1989, pp. 35 a 64 . 
tiva, más dialogante, más negociadora, huyendo de viejas posturas en las que se encastillaba y desde las que administraba y mandaba con modos autoritarios que no sirven en las sociedades democráticas de nuestros días.

Hay, pues, un doble juego en la información que va desde la sociedad a la Administración y desde los poderes públicos a los ciudadanos. «La Administración democrática necesita recoger de la sociedad toda la información posible para que los poderes públicos puedan tomar decisiones adecuadas $y$, al mismo tiempo, se necesita que la Administración suministre a los ciudadanos una amplia información sobre su funcionamiento y estructura interna, al objeto de que se puedan dar los presupuestos básicos que permitan garantizar los derechos y libertades fundamentales que se recogen en nuestro ordenamiento constitucional» (7).

Quiere decirse, en consecuencia, que la Administración Pública tiene, de una parte, que satisfacer las exigencias de la colectividad proporcionándola la información suficiente; y tiene, además, que estar ella misma bien informada para que su acción no esté a merced de la pura casualidad sino que, por el contrario, responda a pautas de racionalidad y previsión en la mayor medida posible.

En los procesos de modernización de las Administraciones Públicas de nuestro tiempo, la necesidad de información resulta decisiva para que dichos procesos se lleven a efecto a partir de propuestas planificadoras bien concebidas y diseñadas. Como han recordado Jacques Chevalier y Daniele Loschak, «la preparación racional de la decisión supone, en primer lugar, la recogida metódica del conjunto de elementos que puedan tener una incidencia sobre la formulación de los problemas y la búsqueda de las soluciones»; indicando seguidamente que "el tratamiento de datos consiste en integrarlos y combinarlos a fin de definir una serie de hipótesis alternativas en función de la información disponible, por una parte, y de los diferentes objetivos considerados por otra». Por ello, los dos autores franceses hablan del upapel crucial de la información», que, en su opinión es «aportación de conocimientos que reduce la incertidumbre en la fase preparatoria de la decisión» $y$, además, "directamente medio de acción e instrumento de poder». $\mathrm{Y}$, con referencia especial a la informática, Chevalier y Loschak, tras afirmar que "en la Administración, en particular, la introducción de los ordenadores implica una serie de consecuencias de importancia desigual, pero cuyo impacto no se puede en ningún caso desestimar», apuntan algunas consecuencias significativas a tener en cuenta: primera, el ordenador posibilita «la

(7) «Los sistemas de gestión...». op. cit., p. 9. 
automatización de la gestión contidiana» ya que, al ordenador, se le pueden encomendar ulas tareas de rutina y las operaciones repetitivas», en la medida en que «no implican ninguna elección o ningún poder de apreciación por parte de la Administración»; y segunda, el ordenador, además, «juega también un papel esencial en el proceso decisional propiamente dicho, como soporte técnico indispensable para la preparación racional de las decisiones" y ello en un triple sentido ya que la informática cumple una «misión documental», proporciona la ayuda precisa «para los cálculos destinados a clarificar las decisiones» $y$, por último, interviene en las llamadas "técnicas de simulación», que sirven para prever los resultados de un determinado proyecto o bien comparar las consecuencias posibles derivadas de políticas diferentes (8).

En el ámbito de los recursos humanos, la información cobra una signficación cada vez más acentuada y relevante en base a diversos factores confluyentes entre sí. En primer término, el propio volumen del personal al servicio de las organizaciones sociales que, en cuanto que van desplegando mayores funciones y nuevas actividades, requieren de nuevos colectivos que se incorporen a unas $y$ otras con el fin de conseguir que el aparato oficial no se paralice. La expansión del protagonismo gubernamental determina el paralelo crecimiento burocrático y el lógico aumento de los servidores públicos. En segundo lugar, aunque el desarrollo funcionarial $y$ burocrático tiene lugar en la mayoría de los países, el hecho de que los Estados encuentren dificultades económicas y financieras en su gestión conlleva el que, de alguna manera, intenten controlar o al menos limitar el crecimiento funcionarial que, para muchos, supone una carga y un coste no fáciles de soportar por lo que se ven en la necesidad de reducir plantillas o de amortiguar el impulso ascendente del empleo público. En tercer lugar, las Administraciones Públicas se tornan cada vez más complejas en su composición y estructuración, lo que ocasiona el que la política de personal

(8) CheVAliER, Jacques, y LOSCHAK, Daniele: "Ciencia Administrativa" (II), Instituto Nacional de Administración Pública, Madrid, 1986, pp. 503 a 506.

Sobre la informatización en el sector público, entre muchas obras, pueden consultarse el Informe de Alain Souloumiac, dirigido al Secretario de Estado francés encargado de la Función Pública y de las Reformas Administrativas, titulado "Les perspectives de l'informatique dans l'Administration», publicado por La Documentación Française en 1983 y que consta de 85 pp.; y. también, la Revista Internacional de Ciencias Administrativas, volumen 56, número 4. correspondiente a diciembre de 1989, y que publica varios trabajos sobre la informatización en la Administración Pública de varios países.

Con referencia a España, puede consultarse el libro La informática en la Administración Pública española. Proyecto Reina 88, editado por la Secretaría General Técnica del Ministerio para las Administraciones Públicas en 1989. Como dice la Orden ministerial de 9 de junio de 1988 que aprobó el Plan Reina para la elaboración del censo de recursos informáticos de nuestra Administración, dicho Plan se contempla como la iniciación en el «desarrollo de una verdadera estrategia de gestión de las tecnologías de la información $y$.las comunicaciones en la Administración Pública, integrada en un planteamiento global de modernización administrativa». 
requiera unas técnicas que superen la sencillez, la tosquedad si se quiere, de las de otros tiempos para dar paso a otras que, apoyadas en las tecnologías más avanzadas, sean capaces de dar respuesta a las nuevas necesidades que se presentan en el campo del personal público. En cuarto lugar, el cambio social que sacude a la sociedad actual también se traslada al área administrativa y burocrática la cual, en lugar de conformase con un inmovilismo tan inútil como perjudicial, ha de dar paso a una concepción dinámica de la Función Pública que, dotada de un sentido provisional y futuro, se anticipe a las transformaciones venideras y estén en condiciones de dar respuesta a todas y cada una de ellas. $Y$, por último, en el ámbito de la Función Pública, al igual que en los demás ámbitos de la vida social, se hace imprescindible el que los funcionarios mejoren sus niveles de conocimientos, actualicen sus saberes profesionales, practiquen las nuevas técnicas que impone el progreso, todo lo cual reclama el que las Administraciones «sepan» dónde tienen que actuar para perfeccionar a sus empleados y dónde han de centrar sus esfuerzos de enseñanza y promoción para mejorar intelectualmente, de una manera racional y programada, a todos sus funcionarios y servidores.

Esta serie de factores mencionados, y brevemente descritos, son los que justifican la necesidad de la información en las Administraciones Públicas. Sólo una Administración bien informada puede ser una Administración bien gestionada $y$ dirigida. Lo contrario es confiarse al voluntarismo de los gestores públicos y esperar que las cosas se resuelvan por la vía de la suerte, la oportunidad o la intuición de los que tienen en sus manos el cuidado de la política de personal. Por eso, Jean-Paul Baquiast ha hablado de «la explosión de las tecnologías de la información y de la comunicación» dado que la Administración, al igual que los demás factores sociales y económicos, debe tender a una utilización eficaz de las técnicas informáticas en busca de objetivos más ambiciosos que no se limiten a lo meramente cotidiano y habitual. Para Jean-Paul Baquiast, Secretario General del Comité Interministerial de Informática y de Burótica en la Administración en Francia, "la gestión del día a día, desarrollando normalmente diversos factores, tales como la anualidad presupuestaria, la movilidad de los responsables, la presión de lo cotidiano, no basta. Es preciso, en contra de la facilidad, adoptar, para la modernización de la Administración, $y$ por tanto para enmarcar su informatización, objetivos a medio y largo plazo, que demuestren una gestión estratégica» (9).

(9) BAQUIAST, Jean-Paul: "La modernisation de l'administratıon», Revista "Futuribles», núm. 124, septiembre 1988, pp. 31 y ss. 


\section{DEL PERSONAL ESTATAL}

Dentro del esquema genérico de la Función Pública de un determinado país, el primer dato a investigar es el del volumen de los efectivos que componen la misma. En la actualidad, todos los Estados se esmeran por conocer de la manera más perfecta posible cuál es el número de empleados públicos que trabajan en los diversos niveles de las Administraciones Públicas, porque se trata de un dato que importa fijar con la mayor exactitud posible como punto de partida para otras consideraciones y como arranque para posteriores análisis y estudios.

En 1984, Alejandro Nieto afirmaba, con relación a España, que "por muy sorprendente que parezca, la realidad es que se desconoce el número de servidores del Estado», al tiempo que añadía que "lo único que de ordinario se afirma es que son demasiados, aunque no faltan tampoco opiniones rigurosamente contratrias, que afirman que son escasos» (10). No obstante, esta negativa situación se ha tratado, en los últimos años, de superar a través, sobre todo, de la puesta a punto del Registro Central de Personal. Prueba de ello es la publicación del Boletín Estadístico del citado Registro, correspondiente a.1 de junio de 1990, cuyo contenido ha servido para la elaboración del presente trabajo.

En la Introducción del Boletín, se empieza afirmando que «la transparencia en su actuación debe ser uno de los requisitos básicos por los que debe regirse la Administración», subrayando a continuación que "si esto es así en todas las ramas de su actividad, debe serlo, con mayor motivo, en relación con los medios humanos con los que cuenta para atender las demandas crecientes que día a día se le plantean». Los datos van referidos al personal inscrito en el Registro Central de Personal a 1 de junio de 1990, así como al resto del personal no inscrito en el mismo (el perteneciente a las Administraciones Autónomica y Local, el de las Fuerzas Armadas, el de la Administración de Justicia, el que integra los Cuerpos y Fuerzas de Seguridad del Estado, el estatutario de la Seguridad Social y el laboral al servicio de las Instituciones Sanitarias). A estos colectivos debe añadirse el personal que integra los llamados altos cargos y el de carácter laboral que trabaja en las sociedades estatales y entes del sector público, no formando tampoco parte del ámbito estadístico del Boletín el personal que tiene contrato de trabajo de duración determinada, no superior a seis meses.

El Boletín utiliza, como fuentes de información, además del propio Registro de Personal, los Presupuestos Generales del

(10) Nieto Garcia, Alejandro: La Organización del desgobierno, Editorial Ariel, Barcelona, 1984 , p. 126. 
Estado, la Encuesta de Población Activa y la documentación que elabora la Intervención General de la Administración del Estado. Esto por lo que se refiere al personal al servicio de la Administración del Estado, ya que, para el resto del personal, la información que proporciona procede de las fuentes que va citando puntualmente en sus cuadros y tablas, como son, entre otras la Base de Datos de la Seguridad Social, la Mutualidad Nacional de Previsión de Administración Local (MUNPAL), la Dirección General de Relaciones con la Justicia y el Consejo General del Poder Judicial, las Direcciones Generales de la Policía y de la Guardia Civil, etc.

Combinando estas fuentes informativas, el Boletín del Registro Central de Personal presenta un conjunto de datos importantes de los cuales hemos seleccionado, en primer lugar, los más destacados sobre la totalidad del personal al servicio del sector público que arroja los siguientes resultados:

Personal al servicio del sector público estatal

Personal al servicio de las Comunidades Autónomas

Personal al servicio de la Administración Local TOTAL

\begin{tabular}{|c|c}
\hline Número & Porcentaje \\
\hline 1.326 .329 & 61,1 \\
514.223 & 23,7 \\
329.811 & 15,2 \\
\hline 2.170 .413 & 100,0 \\
\hline
\end{tabular}

Si ahora prodecemos al desglose del personal estatal, con el fin de identificar a sus diversos componentes, obtenemos estas cifras:

Funcionarios

Docentes en centros no universitarios

Personal de Universidades

Personal laboral inscrito en el Registro

Total de personal inscrito en el Registro

Administración de Justicia

Fuerzas y Cuerpos de Seguridad del Estado

Fuerzas Armadas

Personal de Instituciones Sanitarias

Altos cargos

Personal laboral de Defensa

Sociedades estatales

Total de personal no inscrito en el Registro.

TOTAL

\begin{tabular}{|r|r}
\hline \multicolumn{1}{|c|}{ Número } & Porcentaje \\
\hline 209.199 & 15,8 \\
133.900 & 10,1 \\
61.965 & 4,7 \\
109.274 & 8,2 \\
\hline 514.338 & 38,8 \\
30.178 & 2,3 \\
114.321 & 8,6 \\
125.091 & 9,4 \\
147.542 & 11,1 \\
383 & - \\
39.052 & 3,0 \\
355.424 & 26,8 \\
\hline 811.991 & 61,2 \\
\hline 1.326 .329 & 100,0 \\
\hline
\end{tabular}


De este cuadro numérico, interesa subrayar que, en el total del personal estatal, tan sólo 209.199 efectivos son funcionarios propiamente dichos, lo que representa un porcentaje del 15,8 por 100. Ello viene a destruir, en parte, el tópico generalizado de que hay "muchos funcionarios», siendo así que, en el contexto global de la Administración estatal, no llegan al 16 por 100. Asimismo, si nos fijamos en el personal inscrito en el Registro Central de Personal, se advierte que, aproximadamente, de cada tres empleados públicos uno de ellos es laboral, lo que revela un índice notable de laboralización de la Función Pública estatal provocada, sin duda, por la presencia del Estado en actuaciones no meramente burocráticas o administrativas, sino de otra naturaleza (social, económica, financiera, deportiva, etc.); y por la interpretación inicial que se dio al artículo 15 de la Ley de Medidas hasta que el Tribunal Constitucional, en su sentencia de 11 de junio de 1987 , frenó el proceso laboralizador abierto en la Administración española reconduciendo las aguas a su verdadero cauce (11).

Continuando en esta tarea de interpretación de los datos expuestos, si sumamos todo el personal laboral estatal el resultado es de 503.750 efectivos que suponen el 38 por 100 del total; lo que traduce la importancia cuantitativa de estos trabajadores en la Administración del Estado, lo que, a su vez, demuestra el protagonismo creciente de éste como agente económico-social y como sujeto destacado de comportamientos que tienen su reflejo en las áreas más diversas de la realidad social.

Considerando exclusivamente el personal de los Ministerios propiamente dichos, en el total ya expresado de 514.388 efectivos el mayor número de éstos corresponde al Ministerio de Educación y Ciencia con 220.370 personas, de las cuales, como ya se ha indicado, 133.900 ejercen como docentes no universitarios, al que siguen los Ministerios de Transportes, Turismo y Comunicaciones con 78.377 personas (de las cuales 59.189 pertenecen a los servicios de correos y telecomunicación), de Trabajo y Seguridad Social con 52.339 (excluidos del cómputo el personal estatutario de la Seguridad Social y el laboral al servicio de las instituciones sanitarias) y de Economía y Hacienda con 47.395. Como Ministerios de dimensión intermedia aparecen los de Obras Públicas y Urbanismo con 23.000 personas, de Agricultura, Pesca y Alimentación con 16.238, de Interior con 14.502 (no integradas en los Cuerpos y Fuerzas de Seguridad del Estado), de Justicia con

(11) Vid., entre otros, el trabajo de Fernando BERMEJO CABRERO, titulado "Las relaciones de puestos de trabajo y la laboralización de la Función Públican, publicado en la Revista Española de Derecho Administrativo, núm. 62, correspondiente a los meses de abril a junio de 1989, pp. 229 a 241. 
13.267 (excluido el personal de la Administración de Justicia) y de Sanidad y Consumo con 11.934. Finalmente, como Ministerios de dimensión reducida están los de Asuntos Sociales con 8.789 personas, de Asuntos Exteriores con 5.313, de Cultura con 4.854, de Industria y Energía con 3.292, de Administraciones Públicas con 3.052 , de Relaciones con las Cortes con 2.985 y del Portavoz del Gobierno con 267.

\section{EL PERSONAL AUTONOMICO}

Expuestas algunas estadísticas referidas al personal estatal, en cuanto sirven para establecer comparaciones y buscar analogías y diferencias con otros colectivos de personal público, interesa entrar en el análisis del personal que depende de las diecisiete Comunidades Autónomas que integran el Estado español de las Autonomías. En este caso, los datos estadísticos disponibles cobran un especial interés por cuanto ayudan a dimensionar mejor el fenómeno autonómico y valorar el verdadero alcance del proceso de descentralización política y administrativa seguido en nuestro país a raíz de la entrada en vigor de la Constitución de 1978.

Son bien conocidos los problemas que giran en torno a la Función Pública autonómica y que ha estudiado profusamente la doctrina. Entre otros, Baena del Alcázar se ha referido de modo expreso a esta problemática describiéndola en sus diferentes ramificaciones y manifestaciones (12). Por su parte. Castells Arteche ha descrito detalladamente toda la trayectoria seguida para la configuración de las burocracias autonómicas desde su iniciación hasta la promulgación de las Leyes de Función Pública por las respectivas Comunidades Autónomas (13).

La construcción del Estado de las Autonomías ha supuesto, en todo caso, una radical transformación de la distribución de los efectivos de personal, cuya diversas clases ha sido descrita frecuentemente por la doctrina. Entre otros, Julián Alvárez ha señalado las siguientes clases de personal que han pasado a constituir la Función Pública de cada Comunidad Autónoma. Primero, como núcleo más importante, los funcionarios estatales que fueron transferidos junto con los servicios a los que figuraban adscritos. Segundo, los funcionarios estatales que, con arreglo a lo establecido en la LOAÑA, pudieran llegar a integrar los denomina-

(12) BAEnA DEL AlCÁzAR, Mariano: Curso de Ciencia de la Administración, Editorial Tecnos, Madrid, 1985, pp. 284 a 288.

(13) CASTELLS ARTECHE, José Manuel: Proceso de construcción y desarrollo de la Función Pública Autonómica, Instituto Nacional de Administración Pública, Alcalá de Henares (Madrid). 1987, 263 pp. 
dos Cuerpos declarados por dicha Ley como nacionales; hipótesis legal no convertida en realidad hasta la fecha. Tercero, los funcionarios estatales que, sin pertenecer a los citados Cuerpos Nacionales, han pasado a prestar servicio en las Comunidades Autónomas a petición de éstas. Cuarto, los funcionarios que hayan sido reclutados directamente por cada Comunidad Autónoma en virtud de sus propias atribuciones para integrarse en las respectivas Escalas o Cuerpos juntamente con los funcionarios estatales citados en primer lugar de esta enumeración. $Y$ quinto, como contingentes residuales o complementarios, los contratados de colaboración temporal de régimen administrativo, los contratados laborales transferidos por el Estado, los contratados por las propias Comunidades Autónomas y, finalmente, el personal procedente de las Corporaciones Locales, así como el de la Seguridad Social (14).

Todo este personal, tan heterogéneo y diversificado, desde el momento inicial de las transferencias hasta la fecha, de acuerdo con los datos suministrados por las Comunidades Autónomas en el segundo semestre de 1989 aparece computado de la forma siguiente, de mayor a menor, en cada una de las Comunidades Autónomas:

\begin{tabular}{|c|c|}
\hline \multirow{3}{*}{ Andalucía } & Número \\
\hline & 155.143 \\
\hline & 96.518 \\
\hline Valencia .... & 70.207 \\
\hline País Vasco & 40.786 \\
\hline Galicia ....... & 35.276 \\
\hline Canarias & 28.117 \\
\hline Madrid ....... & 25.625 \\
\hline Castilla-León ....... & 15.081 \\
\hline Castilla-La Mancha & 9.093 \\
\hline Extremadura ....... & 6.780 \\
\hline Aragón ..... & 6.685 \\
\hline Navarra & 6.653 \\
\hline Asturias & 6.232 \\
\hline Murcia & 5.187 \\
\hline Cantabria & 2.794 \\
\hline Baleares. & 2.066 \\
\hline La Rioja & 2.030 \\
\hline TOTAL & 514.273 \\
\hline
\end{tabular}

(14) Alvarez Alvarez, Julián: "El personal al servicio de las Comunidades Autónomas: problemas actuales y perspectivas de futuro», en La Función Pública de las Comunidades Autónomas, Generalitat Valenciana, Valencia, 1983, pp. 225 a 230. 
Los datos acabados de exponer, en los que no figuran los referentes a las Policías Autónomas, pueden ser objeto de análisis desagregado para cada uno de los totales autonómicos citados, en los que, según los cuadros que aporta el Boletín Estadístico del Registro Central de Personal, aparecen incluidos estas clases de personal; funcionarios de carrera, interinos, eventuales, personal docente, sanitarios locales, contratados administrativos, personal laboral y personal estatutario teniendo en cuenta, por lo que concierne a este último personal estatutario $y$ al de carácter docente, que no todas las Comunidades Autónomas han recibido transferencias en materia de enseñanza y seguridad social. Fijándonos, como colectivo más representativo, en los funcionários de carrera que prestan servicio en las Comunidades Autónomas, el orden cuantitativo de mayor a menor es como sigue:

\begin{tabular}{|c|c|}
\hline & Número \\
\hline Cataluña & 10.065 \\
\hline Andalucía .. & 8.966 \\
\hline Castilla-León & 6.032 \\
\hline Valencia .... & 5.383 \\
\hline Madrid & 5.034 \\
\hline Navarra & 4.455 \\
\hline Galicia $\ldots \ldots \ldots \ldots$ & 4.038 \\
\hline Castilla-La Mancha & 3.182 \\
\hline Canarias ............ & 2.782 \\
\hline Murcia ... & 2.686 \\
\hline Aragón & 2.408 \\
\hline Asturias ... & 2.062 \\
\hline País Vasco & 1.973 \\
\hline Extremadura & 1.895 \\
\hline Cantabria ... & 1.200 \\
\hline La Rioja. & 892 \\
\hline Baleares & 511 \\
\hline TOTAL & 63.746 \\
\hline
\end{tabular}

Verificando idéntica operación de cómputo escalonado, ahora con relación al personal laboral, se obtiene esta relación de mayor a menor:

\begin{tabular}{|c|c|}
\hline & Número \\
\hline Madrid ... & 19.826 \\
\hline Andalucía & 11.001 \\
\hline Cataluña & 7.423 \\
\hline Canarias & 6.642 \\
\hline País Vasco & 5.449 \\
\hline Valencia .... & 5.329 \\
\hline
\end{tabular}




\begin{tabular}{|c|c|}
\hline 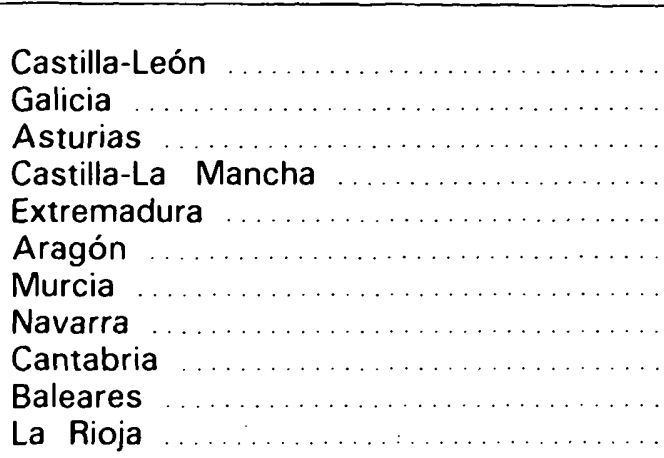 & $\begin{array}{r}\text { Número } \\
4.898 \\
3.903 \\
3.542 \\
3.392 \\
3.022 \\
2.665 \\
1.695 \\
1.672 \\
1.174 \\
843 \\
749 \\
\end{array}$ \\
\hline TOTAL .. & 83.207 \\
\hline
\end{tabular}

Si nos fijamos en las otras clases de personal, se comprueba que, sumando los interinos de todas las Comunidades Autónomas, hay 19.666 funcionarios de esta naturaleza figurando a la cabeza Andalucía con 7.704 interinos cuyo volumen se aproxima mucho al de funcionarios de carrera que son, tal como ya se ha expuesto, 8.966, y Cataluña con 4.509 interinos cuya suma casi representa la mitad de los funcionarios de carrera que son 10.065 en la Comunidad catalana. Los eventuales, lógicamente, tienen un peso específico mucho menor ya que, para todo el conjunto de las diecisiete Comunidades Autónomas, tan sólo ascienden a 910 destacando, de modo especial, la Comunidad Autónoma del País Vasco que tiene 266 y Cataluña que tiene 132, mientras que una Comunidad pequeña como Navarra cuenta con 94 eventuales que es una cifra mayor que la de Comunidades más extensas como Galicia con 85 y Andalucía con 76. Y, por lo que concierne al personal contratado en régimen de Derecho Administrativo, tan sólo aparece computado en algunas Comunidades Autónomas destacando la del País Vasco con 1.260 contratados de un total de 2.136 que es el contingente global llamado a desaparecer de acuerdo con la Disposición Adicional $4 .^{a}$ de la Ley de Medidas.

Ordenando escalonadamente los datos acabados de explicar referentes a determinadas clases de personal, y teniendo a la vista otros que figuran en el Boletín Estadístico y que simplemente hemos enumerado con anterioridad, se puede proponer el siguiente cuadro de las diferentes clases de personal autonómico de mayor a menor:

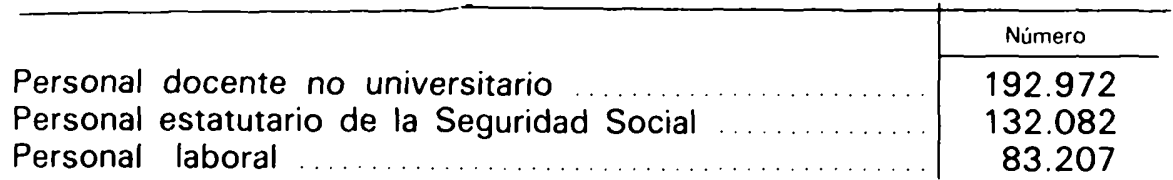


Funcionarios de carrera Interinos

Sanitarios locales

Contratos administrativos

Eventuales

TOTAL

Número

63.746

19.666

19.552

2.136

910

514.273

Procediendo a una agrupación más genérica de los colectivos expuestos, el cuadro anterior se transforma en el siguiente:

\begin{tabular}{|c|c|c|}
\hline & Número & Porcentaje \\
\hline 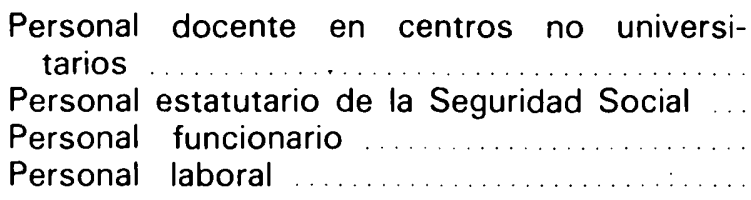 & $\begin{array}{r}192.974 \\
132.082 \\
106.010 \\
83.207\end{array}$ & $\begin{array}{l}37,5 \\
25,7 \\
20,6 \\
16,2\end{array}$ \\
\hline TOTAL & 514.273 & 100,0 \\
\hline
\end{tabular}

A la hora de formular conclusiones que ayuden a interpretar las cifras expuestas y que, de algún modo, contribuyan a subrayar los aspectos más sobresalientes de la Función Pública autonómica, se pueden adelantar las siguientes:

- Destaca la importancia cuantitativa del personal docente que, en el conjunto de servidores públicos autonómicos, representa el 37 por 100 del total, que es tanto como decir que, de cada tres empleados públicos en las Comunidades Autónomas, uno de ellos se inscribe en el sector docente no universitario. $Y$ ello a pesar de que tan sólo cuentan con dicho personal las tres Comunidades históricas, más Andalucía, Canarias y Valencia, además de Navarra.

- El personal funcionario propiamente dicho alcanza el 20 por 100 del total, lo que supone que, de cada cinco servidores públicos autonómicos, uno de ellos pertenece a dicho personal. Los datos manejados no permiten, sin embargo, determinar el grado de "funcionarización» de las Comunidades Autónomas, ya que, para ello, sería preciso establecer el número de funcionarios por cada 1.000 habitantes, 0 , en comparación con la población activa de cada Comunidad o, incluso, con la población del sector servicios. Los números o cifras absolutas que estamos utilizando no revelan ni expresan el nivel de "desarrollo burocrático» de las Comunidades Autónomas. 
- Determinadas Comunidades Autónomas marcan unas cotas elevadas de personal interino destinado en las mismas. Así, en Andalucía, los funcionarios de empleo interinos suman 7.704, mientras que los funcionarios de carrera son tan sólo 8.966, lo que indica una diferencia reducida ciertamente significativa y cuyas causas sería interesante investigar. En Cataluña, los interinos suman 4.509 efectivos, frente a 10.065 funcionarios de carrera. Pero el supuesto más espectacular, no fácilmente interpretable a la vista de los datos presentes, es el del País Vasco, donde los funcionarios interinos, que son 2.290, superan a los funcionarios de carrera, que son 1.973, cifra esta última por lo demás claramente reducida, y que entendemos se debe a que tan sólo se ha computado al personal de la Administración General propiamente dicha, excluyéndose los de las instituciones forales.

- El personal eventual que, según la Ley de Medidas, ejerce «funciones expresamente calificadas de confianza o asesoramiento especial», destaca en las tres Comunidades históricas, así como en Andalucía y también en Navarra y Asturias. Los eventuales del País Vasco, que ascienden a 266, suman casi tantos como los de las otras dos Comunidades históricas y Andalucía, lo que puede prestarse a interpretaciones diversas que van desde un mayor grado de politización de los niveles administrativos superiores a una utilización incorrecta de esta clase de personal.

- Finalmente, en cuanto al personal docente en centros no universitarios, aparece contabilizado en las Comunidades Autónomas que siguieron la vía del artículo 151 de la Constitución; en Navarra y en Canarias y País Valenciano, cuyas competencias se ampliaron a través de la vía prevista en el artículo 150.2 del texto constitucional. Y sobre el personal estatutario de la Seguridad Social, sólo figura reflejado en las Comunidades de Cataluña, País Vasco, Andalucía y Valencia, si bien la de Murcia también aparece con 131 efectivos de esta naturaleza.

\section{EL PERSONAL LOCAL}

Como tercer nivel, después de las Administraciones estatal y autonómica, hay que situar la Administración local que, por lo general, en cuanto a estadísticas y datos, se ha caracterizado entre nosotros por su deficiencia y escasez de unos y otros. El fenómeno se ha agudizado todavía más desde que la autonomía local empezó a caminar por imperativos constitucionales, y los vínculos que, tradicionalmente, unían a las Entidades locales con la Administración del Estado se han ido debilitando cuando no rompiendo $y$ quebrando definitivamente. Precisamente, uno de los ámbitos 
donde más se ha notado este proceso de desvinculación y ruptura es el estadístico, al haberse cerrado, o al menos dificultado, los canales que contribuían a facilitar y obtener información.

De los tres grandes bloques de personal que integran el personal local, cada uno de ellos cuanta con su propia fuente de información lo que, ciertamente, no contribuye a la adecuada homogeneidad y uniformidad requeridas en este tipo de estadísticas. Mientras que los datos referentes a los funcionarios con habilitación de carácter nacional (que el Boletín Estadístico del Registro Central de Personal denomina "Cuerpos») proceden del Registro Central de Personal, los de los funcionarios propios de las Corporaciones Locales han sido extraídos de la MUNPAL y, finalmente, los del personal laboral que trabaja en Ayuntamientos y Diputaciones tienen su origen en la Base de Datos de la Seguridad Social.

A partir de esta triple fuente informativa se puede ofrecer el siguiente cuadro resumen:

\begin{tabular}{|c|c|c|}
\hline & Número & Porcentaje \\
\hline 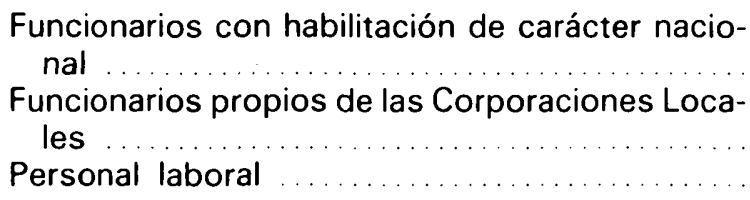 & $\begin{array}{r}5.113 \\
173.096 \\
151.602\end{array}$ & $\begin{array}{r}1,6 \\
\\
52,4 \\
46,0\end{array}$ \\
\hline TOTAL & 329.811 & 100.0 \\
\hline
\end{tabular}

Sobre los funcionarios de Administración Local con habilitación de carácter nacional, el Boletín no aporta ningún dato más, por lo que no es posible proceder a ningún tipo de desglose o pormenorización. $Y$, por lo que concierne al personal funcionario propio de las Corporaciones, el primer desglose a establecer es la diferenciación entre los funcionarios de carrera, que son 164.745, y los funcionarios interinos, que son 8.351. Veamos a continuación con mayor detalle cada uno de estos dos colectivos.

Los funcionarios locales de carrera, si nos atenemos al criterio de la Comunidad Autónoma, donde prestan sus servicios, los podemos ordenar de la forma siguiente:

\begin{tabular}{|c|c|}
\hline & Número \\
\hline Cataluña & 27.177 \\
\hline Andalucía & 27.028 \\
\hline Madrid .. & 21.951 \\
\hline Valencia ... & 17.194 \\
\hline País Vasco & 11.203 \\
\hline
\end{tabular}




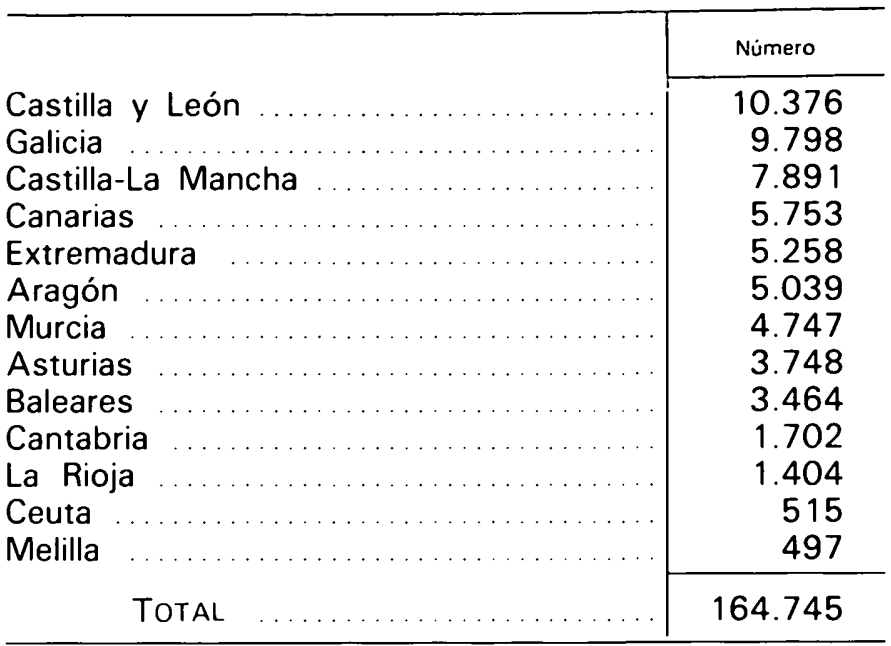

Según los datos de la MUNPAL, las cinco provincias que tienen mayor número de funcionarios locales son las siguientes:

\begin{tabular}{|c|c|}
\hline & Total \\
\hline Barcelona & 22.043 \\
\hline Madrid & 21.951 \\
\hline Valencia & 8.856 \\
\hline Vizcaya & 6.967 \\
\hline Alicante & 6.163 \\
\hline
\end{tabular}

Por el contrario, las cinco provincias que cuentan con un menor número de funcionarios locales son las siguientes:

\begin{tabular}{|c|c|}
\hline & Total \\
\hline Segovia & 778 \\
\hline Cuenca & 765 \\
\hline Huesca & 743 \\
\hline Teruel & 532 \\
\hline Soria & 437 \\
\hline
\end{tabular}

$\mathrm{Si}$ ahora intentamos clasificar al personal funcionario local ateniéndonos al criterio del grupo en el que se inserta, con arreglo 
a la titulación exigida para acceder al mismo, los resultados que se obtienen son éstos:

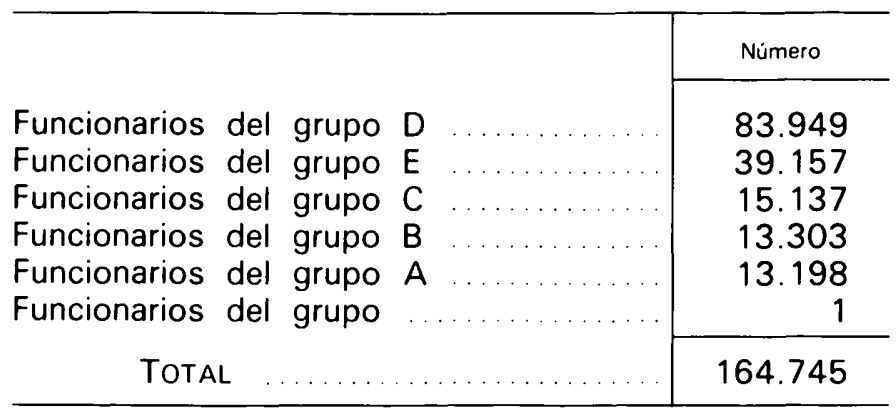

De los funcionarios de empleo interino, cuyo total asciende a 8.351 en nuestra Administración Local, más de la mitad se concentran en tres Comunidades Autónomas, como son Madrid, con 1.809 interinos; Andalucía, con 1.427, y Cataluña, con 1.113. Por el contrario, el menor número de funcionarios interinos se da en las Comunidades Autónomas de Cantabria, con 60; La Rioja, con 42, y Asturias, con 27 . Y si trasladamos e! cómputo al ámbito provincial, las provincias con mayor número de funcionarios interinos son: Madrid, con 1.800; Barcelona, con 848; Valencia, con 534; Pontevedra, con 450, y Sevilla, con 446, y con menor número figuran Soria, Lugo y Orense, con 14; Guadalajara, con 9, y Teruel y Salamanca, con 8.

Si nos fijamos en el grupo en el que se incluye a estos funcionarios interinos, a la cabeza se sitúan los del grupo $D$, con 4.269 efectivos, seguidos de los del grupo $E$, con 1.359; los del grupo $A$, con 1.126; los del grupo $B$, con 1.046, y los del grupo $E$, con 551.

Pasando al otro gran bloque local, el constituido por los trabajadores de las Corporaciones Locales, se agrupan por Comunidades Autónomas, donde están destinados en esta forma:

\begin{tabular}{|c|c|}
\hline & Número \\
\hline Madrid & 37.471 \\
\hline Andalucía & 30.375 \\
\hline Cataluña. & 15.298 \\
\hline Canarias & 11.542 \\
\hline Valencia & 10.207 \\
\hline País Vasco .... & 8.946 \\
\hline Castilla y León .... & 8.802 \\
\hline Castilla-La Mancha & 6.710 \\
\hline Asturias ......... & 4.530 \\
\hline
\end{tabular}




\begin{tabular}{|c|c|}
\hline & Número \\
\hline 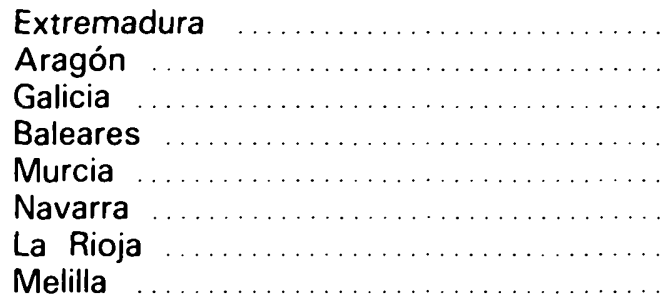 & $\begin{array}{r}3.985 \\
3.706 \\
3.327 \\
3.166 \\
868 \\
704 \\
486 \\
429\end{array}$ \\
\hline TOTAL $\ldots$ & 151.602 \\
\hline
\end{tabular}

A su vez, las cinco provincias que emplean más personal laboral son, de mayor a menor, las siguientes:

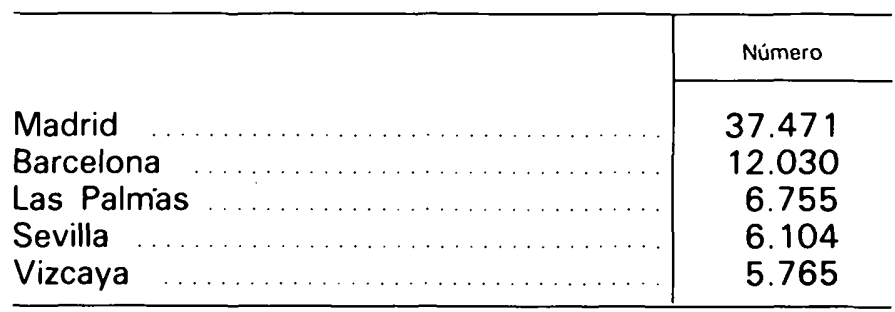

Si contabilizamos ahora las provincias que tienen menos personal laboral, la enumeración es la que sigue:

\begin{tabular}{|c|c|}
\hline & Número \\
\hline Zamora & 353 \\
\hline Lugo .. & 342 \\
\hline Orense & 313 \\
\hline Soria. & 300 \\
\hline Toledo & 147 \\
\hline
\end{tabular}

Atendiendo a una clasificación de carácter profesional, con arreglo a los grupos de tarifa de la Seguridad Social, se obtiene esta triple agrupación:

\begin{tabular}{|c|c|}
\hline & Número \\
\hline $\begin{array}{l}\text { Diversos grupos } \ldots \ldots \ldots \ldots \ldots \\
\text { Peritos y Ayudantes ........... } \\
\text { Ingenieros y Licenciados } \ldots \ldots\end{array}$ & $\begin{array}{r}138.983 \\
7.347 \\
5.272 \\
\end{array}$ \\
\hline TOTAL & 151.602 \\
\hline
\end{tabular}


El mayor número de Ingenieros y Licenciados se concentra en las provincias de Barcelona, con 796; Madrid, con 612, y Vizcaya, con 447. E idéntica concentración se da en cuanto a Peritos y Ayudantes, figurando primero Barcelona, con 866; seguida también de Madrid, con 822, y Vizcaya, con 462. Y en cuanto a otros colectivos incluidos en los restantes grupos de tarifa de la Seguridad Social, Madrid, con 36.037, se destaca sobre las demás provincias, ya que la siguiente, Barcelona, tan sólo cuenta con 10.368, y otras como Las Palmas, Sevilla y Málaga, que aparecen a continuación, tienen, respectivamente, $6.389,5.475$ y 5.239 trabajadores locales.

Si se pretende proponer conclusiones finales, a la vista de los datos expuestos sobre el personal de la Administración Local española, cabe presentar algunas de especial interés y significación como éstas:

- El origen de los datos manejados no es el mismo en todos los colectivos analizados, lo que contribuye a devaluar la fiabilidad de las estadísticas disponibles. Así, por ejemplo, en el cómputo del personal propio de las Corporaciones Locales no se cita a Navarra, cuyas respectivas columnas aparecen en blanco, mientras que dicha Comunidad Autónoma sí está contabilizada en el cuadro del personal laboral. Todo ello denota un problema grave de homogeneidad estadística que, en el futuro, debería resolverse a fin de suprimir la deficiente información estadística de nuestras Entidades Locales.

- Resalta sobremanera la laboralización creciente de nuestros Ayuntamientos y Diputaciones, ya que, de acuerdo con los datos estudiados, los funcionarios propios de las Corporaciones Locales, de carrera e interinos, representan el 52,4 por 100 , que es un porcentaje no muy distante del 46 por 100 que corresponde al personal laboral local. Ello puede deberse a dos causas principales: la primera, el proceso de laboralización que muchas Corporaciones han venido siguiendo en estos últimos años al amparo de una interpretación no siempre correcta de la legislación aplicable, y la segunda, la proyección de la actividad local hacia áreas sociales, económicas, culturales, deportivas, de ocio, etc., lo que reclama necesariamente el reclutamiento de personal sometido al Derecho Laboral en proporciones cada vez más ascendentes.

- Tanto Madrid como Barcelona tienen un gran peso específico en la conformación de la burocracia local española, debido al hecho determinante de que ambas provincias están regidas por dos grandes metrópolis que dan ocupación a importantes colectivos. Madrid, como provincia, emplea más funcionarios locales que La Rioja, Asturias, Cantabria, Baleares, Murcia y Aragón juntas, y aún 
asi, en cuanto al número de dichos funcionarios, es superada por Barcelona. Pero si nos fijamos en el personal laboral, la diferencia a favor de Madrid respecto a las demás provincias es más acentuada, e incluso en comparación con Barcelona, puesto que, aproximadamente, uno de cada cuatro trabajadores locales es madrileño, lo que es un porcentaje ciertamente alto y revelador.

- En lo que concierne a los niveles de titulación, los datos demuestran que el personal laboral es profesionalmente de nivel inferior respecto del personal funcionario. Los funcionarios dei grupo A suponen el 8 por 100 total del colectivo funcionarial, mientras que los trabajadores que son Ingenieros o Licenciados suponen tan sólo el 3,4 por 100 del colectivo laboral. Asimismo, los funcionarios de los grupos C, D y E representan el 83,9 por 100 del total funcionarial, mientras que los trabajadores incluidos en los demás grupos de tarifa de la Seguridad Social (no correspondientes a Ingenieros o Licenciados y a Peritos o Ayudantes) representan el 91 por 100 del total de los trabajadores al servicio de las Entidades Locales.

\section{REFLEXIONES FINALES}

Dos tipos de reflexiones cierran este trabajo, que no tiene más pretensiones que las de divulgar unos datos que se estiman valiosos, dentro de sus evidentes limitaciones, para profundizar en el conocimiento de nuestra Función Pública.

De una parte, a medida que se investiga más en estos temas de la burocracia pública, se hace más patente la trascendencia que, en las Administraciones Públicas tienen las tecnologías informáticas como medios que conducen a incrementar la información acerca del elemento humano en las organizaciones oficiales. Si, como ha escrito Paul $H$. A. Frissen, uen la sociedad de la información, de acuerdo con Bell (1979), la información será el interés central» (15); quiere decirse que, en el campo funcionarial y burocrático, el conocimiento tendrá en el porvenir una relevancia extraordinaria para la gestión racional y ordenada de los recursos humanos.

De otra parte, por lo que afecta al ámbito concreto de la Administración Local y Autonómica, se hace preciso con urgencia ir a un perfeccionamiento de los métodos de investigación, estudio y análisis de los colectivos humanos que trabajan en una y otra. La Administración autonómica está todavía en fase de conformación, puesto que el proceso de nuevas transferencias no está, ni mucho

(15) FRISSEN, Paul H. A.: "La cultura de la informatización», Revista internacional de Ciencias Administrativas, volumen 56, núm. 4, diciembre 1989, p. 42. 
menos, cerrado; $y$, por lo tanto, es previsible que, en años venideros, contingentes de personal sean trasvasados a las Comunidades Autónomas, con lo que se alterarán notablemente las cifras ahora aceptadas, en campos tan decisivos como la enseñanza, la seguridad social, etc. Los datos utilizados sobre personal autonómico no invitan, por el momento, a formular grandes conclusiones, como no sea la de la propia importancia y significación del fenómeno autonómico, que ha permitido que más de medio millón de servidores públicos pasen a ser gestionados por una Administración de nuevo cuño, con lo que tal gestión conlleva de complejidad, distribución del gasto público, reparto de competencias, etc. $\mathrm{Si}$, finalmente, observamos la Administración Local, de evidente arraigo político y social en nuestro país, descubrimos que permanece todavía en una peligrosa penumbra estadística que no permite moverse con pie firme a la hora de combinar datos $y$ valorar estadísticas. Esta es una cuestión de envergadura que debiera ser afrontada por los Poderes públicos buscando soluciones que contribuyan a una clarificación de la vida local en muchas de sus facetas, entre las que destaca la relativa al elemento humano de la misma. No parece, en este sentido, muy de recibo que para calcular los funcionarios propios de nuestras Corporaciones Locales se tenga que acudir, como vía más aprovechable, a los datos de la MUNPAL, porque no hay en funcionamiento otras vías de información más específicas $y$ apropiadas a la finalidad que se pretende. $Y$ si pasamos del orden meramente estadístico al aspecto esencial de la Función Pública Local, está demostrado que, en España, la Administración Local aún no ha alcanzado el desarrollo que tiene en otros países ni en el plano cuantitativo ni en el plano político. Como se ha indicado ya, en el marco global del Estado español, el personal local representa tán sólo el 15,2 por 100 , que es muy inferior al de otros países europeos en los que el porcentaje se eleva al 25 por 100 o incluso al 30 por 100 del total de personal público.

Todo ello parece señalar que, en los próximos años, la distribución del personal público deberá sufrir profundas correcciones en el sentido de que, paralelamente a una disminución del personal estatal, se habrá de producir un aumento significativo del personal autonómico y, sobre todo, un incremento rápido y muy acusado del personal local, especialmente en sus empleados y agentes sometidos al Derecho Laboral. De esta manera, asistiremos al inexorable reequilibrio de nuestras burocracias públicas, hoy demasiado escoradas hacia el lado estatal, y precisadas de un fuerte reajuste que las potencie y mejore en los ámbitos autonómico y local. 